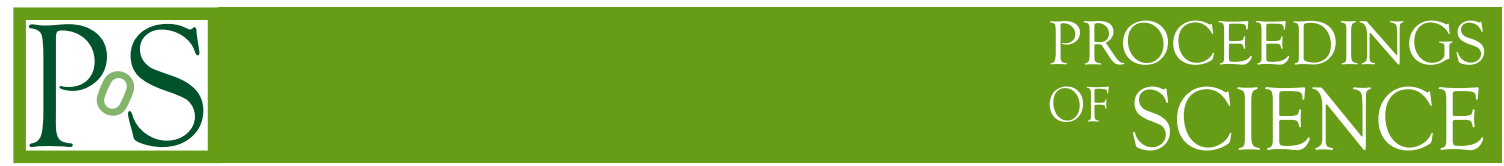

\title{
Lepton universality tests in kaon decays at NA62
}

\section{Francesca Bucci ${ }^{* \dagger}$}

INFN, Sezione di Firenze

E-mail: francesca.bucciefi.infn.it

\begin{abstract}
A precision measurement of the helicity-suppressed ratio $R_{K}=\Gamma\left(K^{ \pm} \rightarrow e^{ \pm} v\right) / \Gamma\left(K^{ \pm} \rightarrow \mu^{ \pm} v\right)$ has been performed using the full dedicated data sample collected by the NA62 experiment at CERN in 2007-2008. The result, $R_{K}=(2.488 \pm 0.010) \times 10^{-5}$, is in agreement with the Standard Model expectation and allows to test the lepton flavor universality with the same accuracy as the tau and pion leptonic decays.
\end{abstract}

The XIth International Conference on Heavy Quarks and Leptons,

June 11-15, 2012

Prague, Czech Republic

${ }^{*}$ Speaker.
†On behalf of the NA62 collaboration 


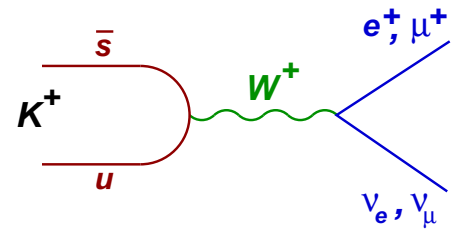

(a)

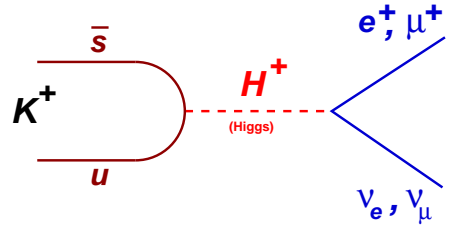

(b)

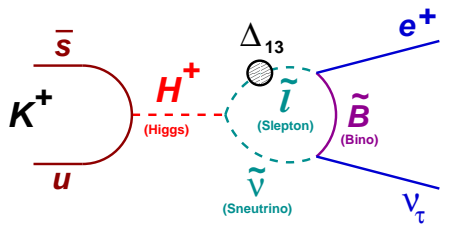

(c)

Figure 1: (a) The SM contribution to the kaon leptonic decays. (b) $H^{ \pm}$exchange in two-Higgs-doublets models at tree level. (c) $H^{ \pm}$exchange in two-Higgs-doublets models at one-loop level.

\section{Introduction}

High precision tests of the lepton flavor universality (LFU) are a powerful tool to probe the Standard Model (SM) fully complementary to the measurements of flavor changing neutral current (FCNC) decays and to the direct exploration of possible new physics (NP) at the Large Hadron Collider (LHC).

In the SM, the leptonic kaon decays $K^{ \pm} \rightarrow e^{ \pm} v\left(K_{e 2}\right)$ and $K^{ \pm} \rightarrow \mu^{ \pm} v\left(K_{\mu 2}\right)$ are mediated by a charged current at tree level (see Fig. 1(a)). The size of non-SM contributions depends on the particular beyond of SM (BSM) scenario we consider. In various frameworks the effect is within the present experimental precision but hadronic uncertainties prevent us from fully exploiting such decays in constraining NP. However, in the $R_{K}$ ratio the hadronic uncertainties cancel to a very large extent and the SM prediction [1] is known with excellent accuracy: $R_{K}(\mathrm{SM})=(2.477 \pm 0.001) \times$ $10^{-5}$.

To obtain accurate predictions, the radiative process $K^{ \pm} \rightarrow e^{ \pm} v \gamma\left(K_{e 2 \gamma}\right)$ must be included. In $K_{e 2 \gamma}$ photons can be produced via internal bremmstrahlung (IB) or direct emission, the latter being dependent on the hadronic structure (Structure Dependent component, SD). By definition, the theoretical prediction of $R_{K}$ is inclusive of the IB radiation only. Therefore, to compare data with the SM prediction at the \%o level, the SD contribution must be carefully estimated and subtracted. The same arguments apply in principle to $K^{ \pm} \rightarrow \mu^{ \pm} v$. However, in this case there is no helicity suppression and the SD contribution can be safely neglected.

Charged Higgs bosons appearing in any model with two Higgs doublets can contribute at tree level (see Fig. 1(b)). Such tree level contribution does not introduce any lepton flavor dependent correction, thus it does not affect the ratio $R_{K}$. The first SUSY contributions violating the $\mu$-e universality in $K^{ \pm} \rightarrow l^{ \pm} v$ decays arise at one loop level (see Fig. 1(c)). In particular, lepton flavor violating (LFV) couplings can contribute to $R_{K}$ at $\%$ level for reasonable SUSY parameters [2] ( $m_{H} \sim 500 \mathrm{GeV}, \tan \beta=40$ and $\Delta_{R}^{31} \sim 10^{-3}$, where $\Delta_{R}^{31}$ is the mixing parameter between the righthanded superpartners of leptons). Actually, the very recent limits on $B_{s} \rightarrow \mu^{+} \mu^{-}$[3] might severely constrain the allowed regions in SUSY parameters space for large $\tan \beta$ [4].

\section{Beam, detector and data taking}

The NA62 experiment at the CERN Super Proton Syncrotron (SPS) collected a dedicated data sample during 2007 and 2008 aiming at a measurement of $R_{K}$ with a $0.4 \%$ precision. For the $R_{K}$ 


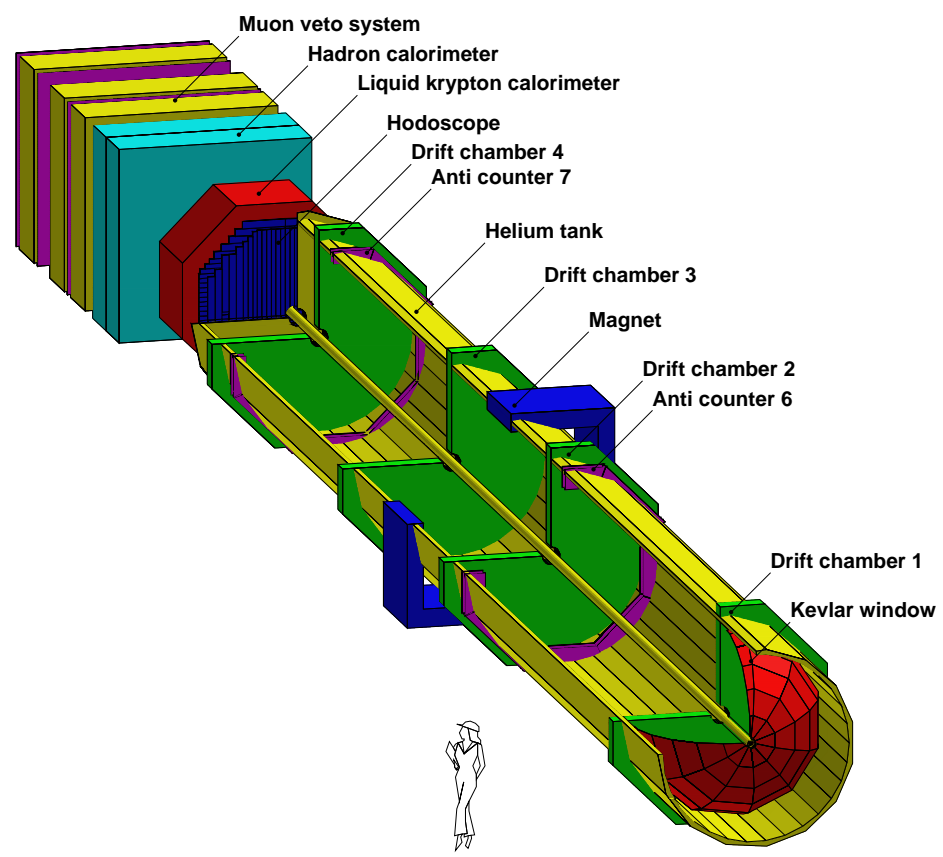

Figure 2: The NA48/2 experimental layout.

measurement, the beam line and setup of the earlier NA48/2 experiment were used [5]. Unseparated secondary charged hadronic beams with central momentum of $74 \mathrm{GeV} / \mathrm{c}$ and momentum spread of $\pm 1.4 \mathrm{GeV} / \mathrm{c}$ were derived from the primary $400 \mathrm{GeV} / \mathrm{c}$ protons extracted from the SPS and impinging on a berillium target. The beam composition was dominated by pions with a kaon fraction of about $6 \%$.

The NA48/2 experimental layout is shown in Fig. 2. The momenta of the charged decay products were measured by a magnetic spectrometer housed in a tank filled with helium at nearly atmospheric pressure and placed after the decay volume. The spectrometer comprised four drift chambers (DCHs) and a dipole magnet placed between the second and the third chamber. A counter hodoscope (HOD) consisting of two planes of plastic scintillators produced fast trigger signals and provided precise time measurements $\left(\sigma_{t} \sim 150 \mathrm{ps}\right)$. A liquid krypton electromagnetic calorimeter $(\mathrm{LKr})$ located further downstream was used for lepton identification and as photon veto detector.

The data taking took place during four months in 2007 and two weeks in 2008 and was optimized to measure the two main backgrounds in the $K_{e 2}$ sample due to the intense flux of muons accompanying the hadronic beam (beam halo muons) and to the $K_{\mu 2}$ decays with a muon misidentified as an electron. Due to the different acceptance and background conditions, $K^{+}$and $K^{-}$ decays as well as data collected with and without the lead wall, as explained later on in Sec. 3.2, were analyzed separately. 


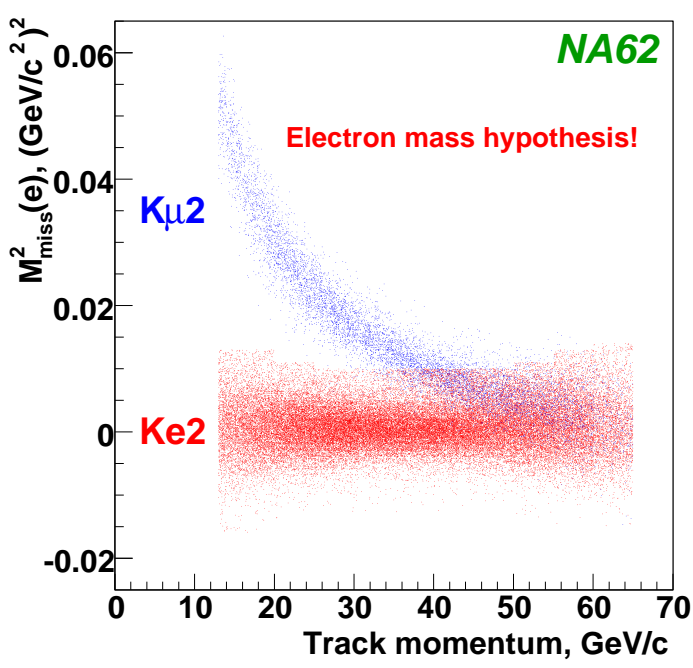

(a)

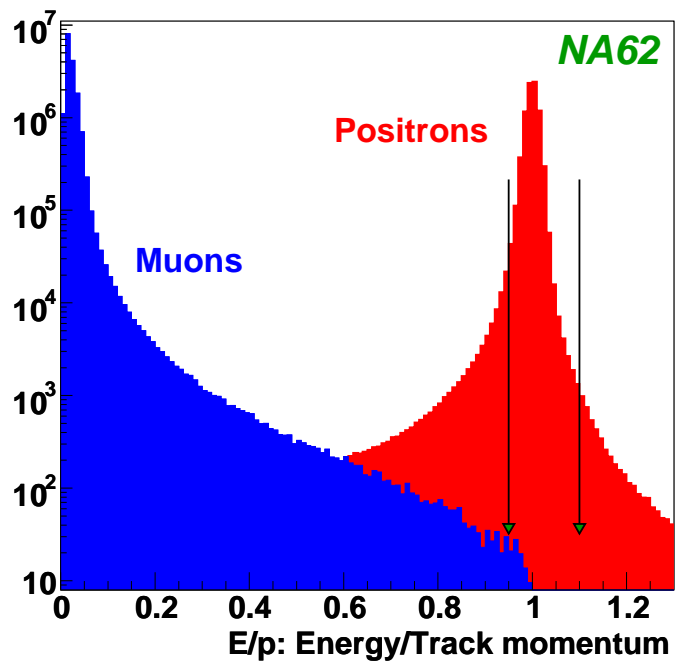

(b)

Figure 3: (a) Squared missing mass in electron mass hypothesis as a function of lepton momentum for reconstructed $K_{e 2}$ and $K_{\mu 2}$ decays. Kinematic separation is achievable at low momentum only. (b) E/p spectra of electrons and muons.

\section{3. $R_{K}$ measurement}

\subsection{Analysis strategy}

The analysis strategy was based on counting the number of reconstructed $K_{e 2}$ and $K_{\mu 2}$ candidates concurrently collected. Consequently, the analysis did not rely on an absolute beam flux measurement, and several systematic effects cancelled at first order. MC simulation was only used to estimate the acceptance correction and the geometrical part of the acceptances for most background processes while particle identification, trigger, read out efficiencies and muon halo background were directly measured using data. Due to the significant dependence of acceptance and backgrounds on lepton momentum, the $R_{K}$ measurement was performed independently in ten bins of momentum.

A minimum-bias trigger configuration was used, resulting in high efficiency with relatively low purity. The $K_{e 2}$ trigger condition consisted of :

- the coincidence of hits in the HOD planes (Q1 signal);

- loose lower and upper limits on multiplicity of hits in the DCHs (1 track signal);

- $\mathrm{LKr}$ energy deposit of at least $10 \mathrm{GeV}$ (ELKr signal).

The $K_{\mu 2}$ trigger condition required a coincidence of the Q1 and 1 track signals down-scaled by a factor $\mathrm{D}=150^{1}$.

\footnotetext{
${ }^{1}$ The non down-scaled $K_{\mu 2}$ trigger rate is $0.5 \mathrm{MHz}$, and is dominated by beam halo muons. The $K_{e 2}$ trigger rate is $\sim 10 \mathrm{kHz}$.
} 


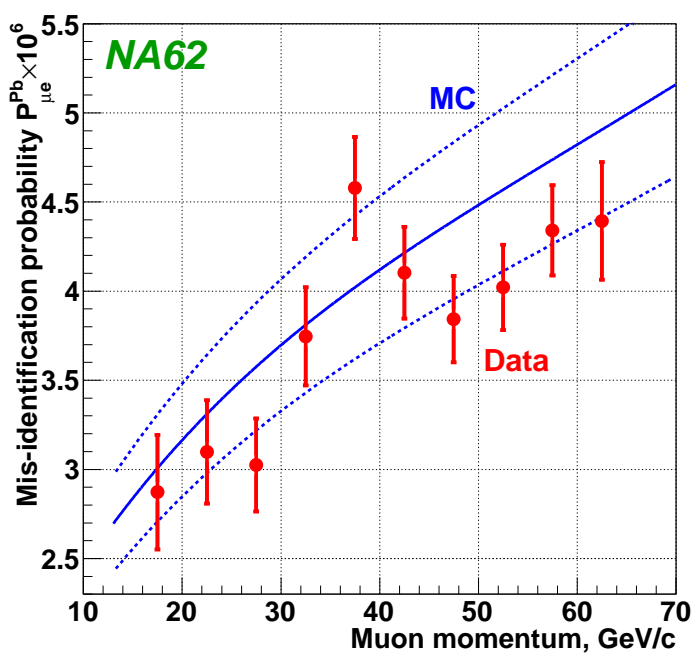

(a)

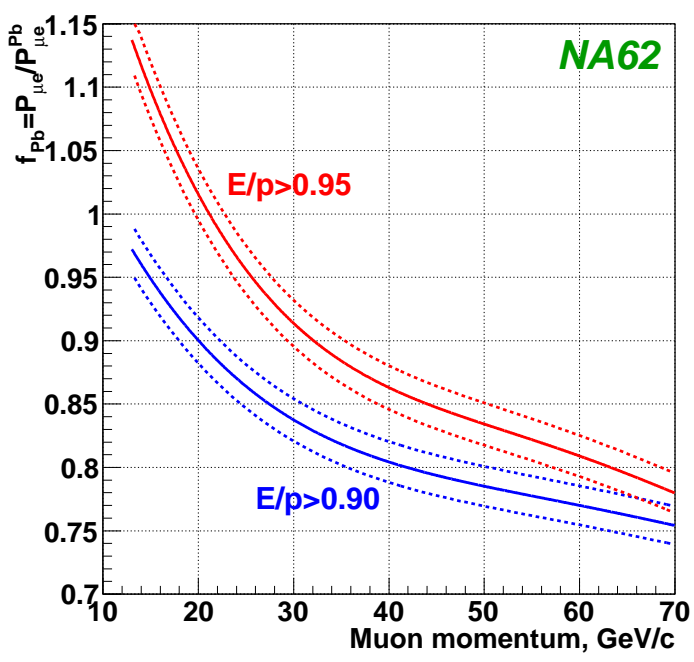

(b)

Figure 4: (a) Mis-identification probability for muon traversing the lead wall as a function of track momentum; (b) Correction factors as evaluated with simulation.

Due to the topological similarity of $K_{e 2}$ and $K_{\mu 2}$ decays, a large part of the selection was in common for the two decay modes: a single charged track with momentum between 13 and $65 \mathrm{GeV} / \mathrm{c}$ was required; a cut on the kaon vertex position, reconstructed as the point of closest approach of the lepton candidate track and the kaon beam axis, was applied to remove the bulk of the beam halo background; photons are vetoed to suppress backgrounds from $K^{ \pm} \rightarrow e^{ \pm} v \gamma$, $K^{ \pm} \rightarrow \pi^{0} e^{ \pm} v, K^{ \pm} \rightarrow \pi^{0} \pi^{ \pm}$decays.

Then, to distinguish between $K_{e 2}$ and $K_{\mu 2}$ decays, two main criteria were used :

- the kinematic identification based on the reconstructed squared missing mass, $M_{m i s s}^{2}(l)=$ $\left(P_{K}-P_{l}\right)^{2}$, where $P_{K}$ and $P_{l}(l=e, \mu)$ are the four-momenta of the kaon and lepton, respectively (see Fig. 3(a));

- and the lepton identification based on the ratio of the energy deposition in the LKr over the momentum measured by the spectrometer (see Fig. 3(b)).

\subsection{Backgrounds}

At high momentum, the $K_{\mu 2}$ decay is the largest background source to $K_{e 2}$. The dominant process leading to mis-identify a muon as an electron is bremmstrahlung in or in front of the LKr with a significant energy deposit in the LKr. The muon mis-identification probability $P_{\mu e}$ was measured using data. To collect a muon sample free from electron contamination due to muon decays, a 9.2 $X_{0}$ thick lead wall covering $20 \%$ of the geometrical acceptance was installed in front of the calorimeter between the two planes of the HOD during a fraction of the data taking. However, the muon passage through the lead wall affects the muon mis-identification probability $P_{\mu e}$ via two principal mechanisms: muon energy loss by ionization, dominating at low momentum; bremmstrahlung, dominating at high momentum. To evaluate the correction factors, $f_{P b}=P_{\mu e} / P_{\mu e}^{P b}$, a dedicated MC 


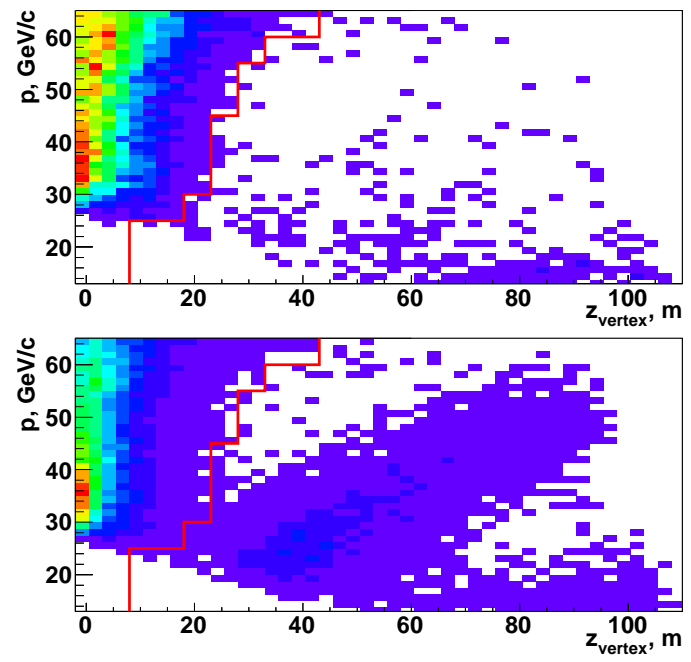

(a)

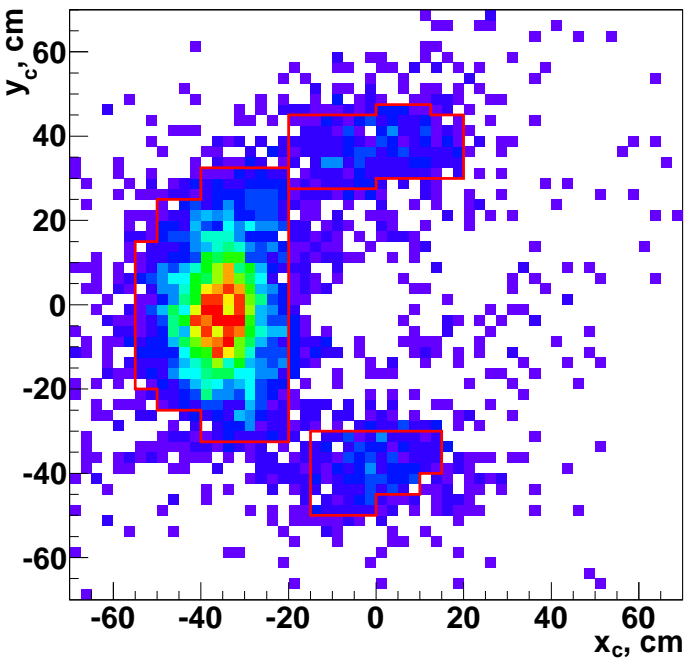

(b)

Figure 5: (a) Reconstructed positron (top) and electron (bottom) candidate momentum as a function of the reconstructed longitudinal coordinate of the kaon decay vertex, $z_{\text {vertex }}$. (b) Distribution of the electron candidate crossing point at the final collimator $(\mathrm{z}=0)$ after applying the cut on $z_{\text {vertex }}$.

simulation based on Geant 4 was developed. The measured muon mis-identification probability as a function of the track momentum compared with MC simulation is shown in Fig. 4(a). The LKr calorimeter reconstruction has been optimized for shower initiated by electrons and photons and starting near its front surface, whereas showers initiated by muon bremmstrahlung start throughout the detector volume. The corresponding relative systematic uncertainty on $P_{\mu e}$ due to energy calibration and cluster reconstruction is of $10 \%$. However, the uncertainty on the ratio $f_{P b}$ is significantly smaller $(\sim 2 \%)$ due to a cancellation of the main systematic effects. The correction factors obtained from simulation along with the estimated systematic uncertainties are shown in Fig. 4(b).

The beam halo muons can become a source of background in case of muon decay or muon mis-identification as an electron. The beam halo background was measured on data: the $K^{+}\left(K^{-}\right)$ only beam was used to measure the muon halo background in the $K^{-} \rightarrow e^{-} \bar{v}\left(K^{+} \rightarrow e^{+} v\right)$ sample, respectively. The probability to reconstruct a $K^{-} \rightarrow e^{-} \bar{v}\left(K^{+} \rightarrow e^{+} v\right)$ candidate due to a decay of an opposite sign kaon, $K^{+}\left(K^{-}\right)$, has been taken into account. Control samples were normalized to data in the squared missing mass region not compatible with a kaon decay. To reduce this background, a cut on the decay vertex longitudinal position was applied. Fig. 5(a) shows the lower cut applied on $z_{\text {vertex }}$ as a function of the momentum (red solid line). The muon sweeping system provided better suppression of the positive beam halo component and the strong charge asymmetry is clearly visible. The residual background in the $K^{-} \rightarrow e^{-} \bar{v}$ sample is 5 times the one in the $K^{+} \rightarrow e^{+} v$ sample. A further beam halo background suppression in the $K^{-} \rightarrow e^{-} \bar{v}$ sample was achieved by applying a cut on the crossing point of the electron candidate at the final collimator plane ( $\mathrm{z}=0$ ). Fig. 5(b) shows the distribution of the crossing point after applying the cut on $z_{\text {vertex }}$.

Due to the $R_{K}$ definition, the SD component of the radiative $K^{ \pm} \rightarrow e^{ \pm} v \gamma$ decay must be carefully estimated and subtracted. The component with positive photon helicity $\left(\mathrm{SD}^{+}\right)$peaks 


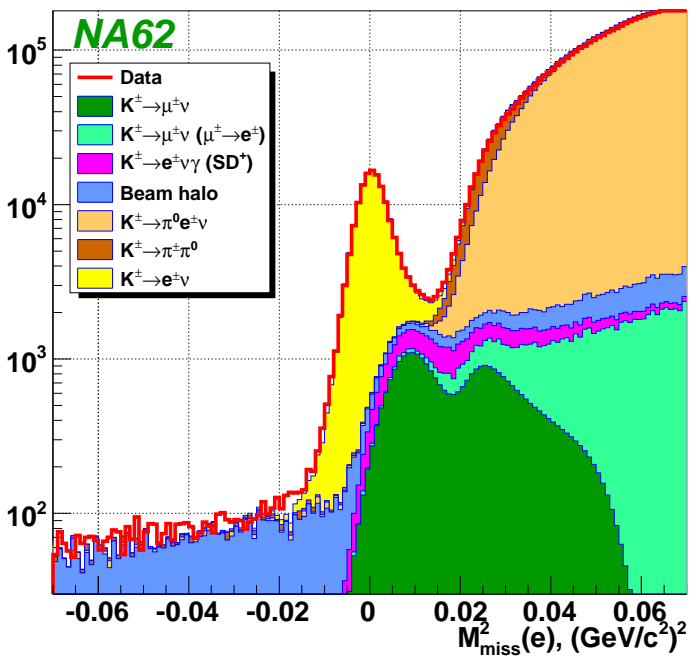

(a)

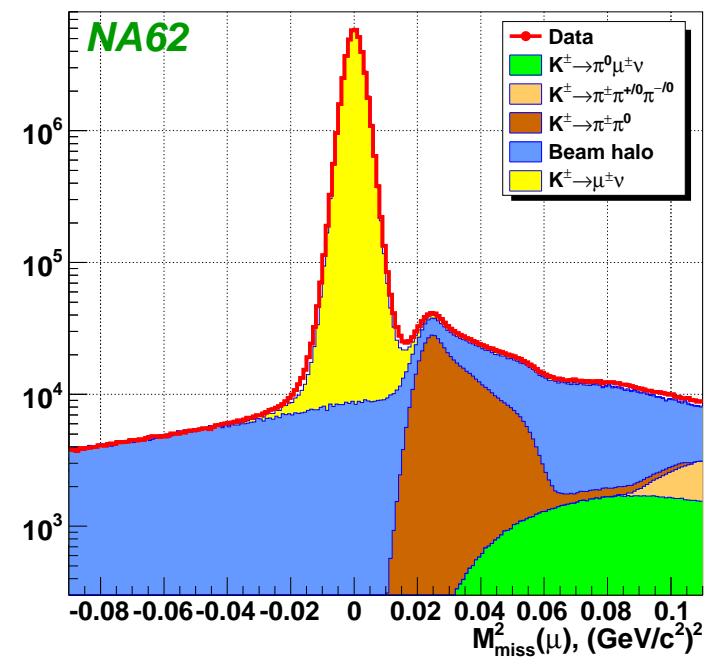

(b)

Figure 6: (a) Reconstructed squared missing mass distribution for the $K_{e 2}$ candidates. (b) Reconstructed squared missing mass distribution for the $K_{\mu 2}$ candidates

at $E_{e}^{*} \sim \frac{M_{K}}{2}$ [6], where $E_{e}^{*}$ is the electron momentum in the kaon rest frame, therefore it is kinematically similar to the $K_{e 2}$ decay. The component with negative photon helicity $\left(\mathrm{SD}^{-}\right)$, peaking at $E_{e}^{*} \sim \frac{M_{K}}{4}$, is kinematically incompatible with the $K_{e 2}$ decay and the corresponding background is negligible. Similarly, the background from the interference between the IB and the SD processes is negligible. The $\mathrm{SD}^{+}$background contribution has been estimated by Monte Carlo simulation using a measurement of the $K^{ \pm} \rightarrow e^{ \pm} v \gamma\left(\mathrm{SD}^{+}\right)$differential decay rate [7].

\section{4. $R_{K}$ results}

The squared missing mass distribution in the electron hypothesis both for data and Monte Carlo is shown in Fig. 6(a). 145,958 $K_{e 2}$ candidates were reconstructed with a total background contamination of $(10.95 \pm 0.27) \%$ in the selected signal region ${ }^{2}$. Background contaminations integrated over the lepton momentum are listed in Table 1.

The squared missing mass distribution in the muon hypothesis both for data and Monte Carlo is shown in Fig. 6(b). $42.817 \times 10^{6} K_{\mu 2}$ candidates have been collected with a down-scaling factor $\mathrm{D}=150$ and the total background contamination in the selected signal region is $(0.50 \pm 0.01) \%$. The only significant source of background in the $K_{\mu 2}$ sample is due to the beam halo and was measured by using the same technique as for the $K_{e 2}$ sample.

The final result was obtained by performing a fit over 40 independent $R_{K}$ measurements ( 4 data samples times 10 momentum bins). The fit result is

$$
R_{K}=\left(2.488 \pm 0.007_{\text {stat. }} \pm 0.007_{\text {syst. }}\right) \times 10^{-5}
$$

with $\chi^{2} /$ ndf $=47 / 39$. The uncertainties are summarized in Table 2 .

\footnotetext{
${ }^{2}$ The lower and upper limits on the squared missing mass vary across lepton momentum bins, taking into account the squared missing mass resolution, radiative mass tails, and background conditions.
} 
Table 1: Background contaminations in the $K_{e 2}$ sample integrated over the lepton momentum.

\begin{tabular}{lc}
\hline Data sample & $\mathrm{B} /(\mathrm{S}+\mathrm{B})$ \\
\hline$K^{ \pm} \rightarrow \mu^{ \pm} v$ & $(5.64 \pm 0.20) \%$ \\
$K^{ \pm} \rightarrow \mu^{ \pm} v(\mu \rightarrow e)$ & $(0.26 \pm 0.03) \%$ \\
$K^{ \pm} \rightarrow e^{ \pm} v \gamma\left(\mathrm{SD}^{+}\right)$ & $(2.60 \pm 0.11) \%$ \\
$K^{ \pm} \rightarrow \pi_{D}^{0} e^{ \pm} v$ & $(0.18 \pm 0.09) \%$ \\
$K^{ \pm} \rightarrow \pi_{D}^{0} \pi^{ \pm}$ & $(0.12 \pm 0.06) \%$ \\
Wrong sign K & $(0.04 \pm 0.02) \%$ \\
Beam halo & $(2.11 \pm 0.09) \%$ \\
\hline Total & $(10.95 \pm 0.27) \%$ \\
\hline
\end{tabular}

Table 2: Summary of the uncertainties on $R_{K}$

\begin{tabular}{lc}
\hline Source & $\delta R_{K} \times 10^{5}$ \\
\hline Statistical & 0.007 \\
\hline$K_{\mu 2}$ background & 0.004 \\
$K^{ \pm} \rightarrow e^{ \pm} v \gamma\left(\mathrm{SD}^{+}\right)$background & 0.002 \\
$K^{ \pm} \rightarrow \pi^{0} e^{ \pm} v, K^{ \pm} \rightarrow \pi^{0} \pi^{ \pm}$backgrounds & 0.003 \\
Muon halo background & 0.002 \\
Matter composition in the spectrometer & 0.003 \\
Acceptance correction & 0.002 \\
Spectrometer alignment & 0.001 \\
Electron identification efficiency & 0.001 \\
1-track trigger efficiency & 0.001 \\
LKr readout inefficiency & 0.001 \\
Total systematic & 0.007 \\
\hline Total & 0.010 \\
\hline
\end{tabular}

The current world average is $R_{K}^{W A}=(2.488 \pm 0.009) \times 10^{-5}$ (see Fig. 7). The value measured by the KLOE collaboration is $R_{K}=(2.493 \pm 0.031) \times 10^{-5}$. They reached a precision of $\sim 1.3 \%$ running over $2.2 \mathrm{fb}^{-1}$ and using a completely different technique [7].

\section{Conclusions}

The NA62 collaboration performed the measurement of $R_{K}$ on the full data sample collected in 2007-2008. The result, $R_{K}=(2.488 \pm 0.010) \times 10^{-5}$, is in agreement with the SM expectation at $1.2 \sigma$. The precision achieved, $4 \%$, allows to test the lepton flavor universality with the same accuracy as the tau and pion leptonic decays [8]. Within the NA62 experimental program [9], the precision on the $R_{K}$ measurement can be further improved because the hermetic photon veto will strongly decrease the $K^{ \pm} \rightarrow e^{ \pm} v \gamma$ background and the beam spectrometer, allowing time correlation between kaons and decay products, will reduce the beam halo background to negligible levels. 


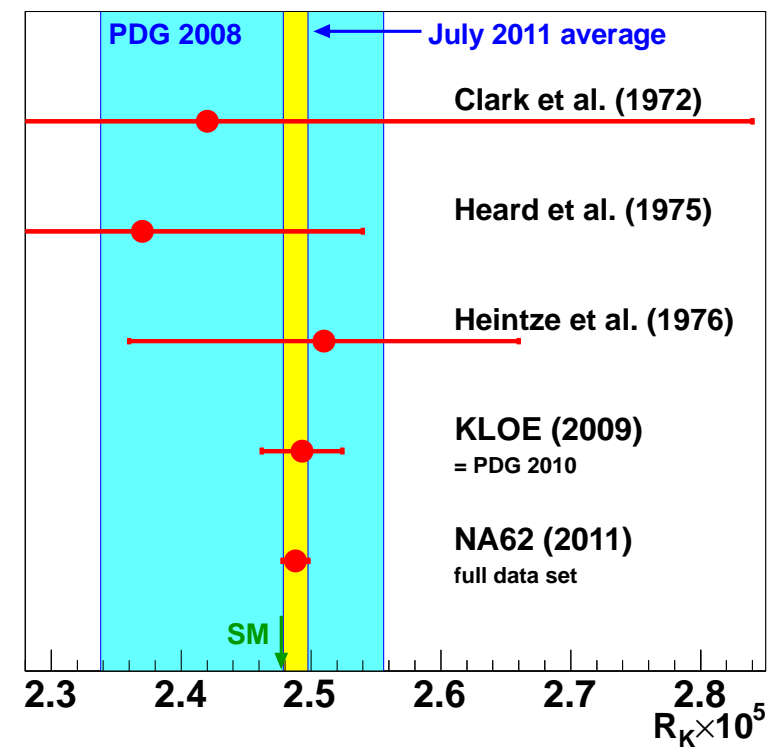

Figure 7: The development of the $R_{K}$ measurement.

\section{References}

[1] V. Cirigliano and I. Rosell, Phys. Rev. Lett. 99, 231801 (2007).

[2] A. Masiero, P. Paradisi and P. Petronzio, Phys. Rev. D 74, 0011701 (2006).

[3] R. Aaij et al. [LHCb Collaboration] arXiv:1203.4493 [hep-ex].

[4] R. Fonseca, J.C. Romão, A. M. Teixiera, arXiv:1250.1411 [hep-ph].

[5] V. Fanti et al., Nucl. Instrum. Meth. A 574433 (2007).

[6] J. Bijens, G. Ecker and J. Gasser, Nucl. Phys B 39681 (1993).

[7] F. Ambrosino et al., Eur. Phys. J. C 64627 (2009). Erratum-ibid. Eur. Phys. J. C 65703 (2010).

[8] A. Pich, XI ${ }^{\text {th }}$ International Conference on Heavy Quarks and Leptons 2012.

[9] G. Anelli et al., CERN-SPSC-2005-013, SPSC-P326. 